\title{
Certain Properties of Multivalent Functions Associated with the Dziok-Srivastava Operator
}

\author{
T. M. Seoudy \\ Department of Mathematics, Faculty of Science, Fayoum 63514, Egypt \\ Correspondence should be addressed to T. M. Seoudy; tms00@fayoum.edu.eg
}

Received 11 October 2012; Accepted 26 October 2012

Academic Editor: Harold Benson

Copyright (C) 2013 T. M. Seoudy. This is an open access article distributed under the Creative Commons Attribution License, which permits unrestricted use, distribution, and reproduction in any medium, provided the original work is properly cited.

By making use of the techniques of the differential subordination, we derive certain properties of $p$-valent functions associated with the Dziok-Srivastava operator.

\section{Introduction}

Let $A(p, k)$ denote the class of functions of the form

$$
f(z)=z^{p}+\sum_{n=k}^{\infty} a_{n+p} z^{n+p} \quad(p, k \in \mathbb{N}=\{1,2,3, \ldots\}),
$$

which are analytic in the open unit disk $U=\{z \in \mathbb{C}:|z|<$ $1\}$. We write $A(p, 1)=A(p)$.

Suppose that $f$ and $g$ are analytic in $U$. We say that the function $f$ is subordinate to $g$ in $U$, or $g$ superordinate to $f$ in $U$, and we write $f<g$ or $f(z) \prec g(z)(z \in U)$, if there exists an analytic function $\omega$ in $U$ with $\omega(0)=0$ and $|\omega(z)|<1$, such that $f(z)=g(\omega(z))(z \in U)$. If $g$ is univalent in $U$, then the following equivalence relationship holds true (see [1-3]):

$$
f(z) \prec g(z) \Longleftrightarrow f(0)=g(0), \quad f(U) \subset g(U) .
$$

For functions $f_{j} \in A(p, k)$ given by

$$
f_{j}(z)=z^{p}+\sum_{n=k}^{\infty} a_{n+p, j} z^{n+p} \quad(j=1,2 ; p \in \mathbb{N}),
$$

we define the Hadamard product (or convolution) of $f_{1}$ and $f_{2}$ by

$$
\left(f_{1} * f_{2}\right)(z)=z^{p}+\sum_{n=k}^{\infty} a_{n+p, 1} a_{n+p, 2} z^{n+p}=\left(f_{2} * f_{1}\right)(z)
$$

For complex parameters $a_{1}, \ldots, a_{q}$ and $b_{1}, \ldots, b_{s}(b j \notin$ $\left.\mathbb{Z}_{0}^{-}=\{0,-1,-2, \ldots\} ; j=1, \ldots, s\right)$, the generalized hypergeometric function ${ }_{q} F_{s}$ is defined (see [4]) by the following infinite series:

$$
\begin{array}{r}
{ }_{q} F_{s}\left(a_{1}, \ldots, a_{i}, \ldots, a_{q} ; b_{1}, \ldots, b_{s} ; z\right)=\sum_{n=0}^{\infty} \frac{\left(a_{1}\right)_{n} \cdots\left(a_{q}\right)_{n}}{\left(b_{1}\right)_{n} \cdots\left(b_{s}\right)_{n}} \frac{z^{n}}{n !} \\
\left(q \leq s+1 ; q, s \in \mathbb{N}_{0}=\mathbb{N} \cup\{0\} ; z \in U\right),
\end{array}
$$

where $(\theta)_{n}$ is the Pochhammer symbol defined, in terms of the Gamma function $\Gamma$, by

$$
(\theta)_{n}=\frac{\Gamma(\theta+n)}{\Gamma(\theta)}= \begin{cases}1, & (v=0), \\ \theta(\theta+1) \cdots(\theta+n-1), & (v \in \mathbb{N}) .\end{cases}
$$

Corresponding a function $h_{p}\left(a_{1}, \ldots, a_{i}, \ldots, a_{q} ; b_{1}, \ldots, b_{s} ; z\right)$ defined by

$$
\begin{aligned}
& h_{p}\left(a_{1}, \ldots, a_{i}, \ldots, a_{q} ; b_{1}, \ldots, b_{s} ; z\right) \\
& \quad=z^{p} \cdot{ }_{q} F_{s}\left(a_{1}, \ldots, a_{i}, \ldots, a_{q} ; b_{1}, \ldots, b_{s} ; z\right) \quad(z \in U),
\end{aligned}
$$

Dziok and Srivastava [5] considered a linear operator

$$
H_{p}\left(a_{1}, \ldots, a_{q} ; b_{1}, \ldots, b_{s}\right): A(p, k) \longrightarrow A(p, k)
$$


defined by the following Hadamard product:

$$
\begin{array}{r}
H_{p}\left(a_{1}, \ldots, a_{q} ; b_{1}, \ldots, b_{s}\right) f(z), \\
=h_{p}\left(a_{1}, \ldots, a_{i}, \ldots, a_{q} ; b_{1}, \ldots, b_{s} ; z\right) * f(z), \\
\quad\left(q \leq s+1 ; q, s \in \mathbb{N}_{0} ; z \in U\right) .
\end{array}
$$

If $f \in A(p, k)$ is given by $(1)$, then we have

$$
\begin{aligned}
& H_{p}\left(a_{1}, \ldots, a_{q} ; b_{1}, \ldots, b_{s}\right) f(z) \\
& =z^{p}+\sum_{n=k}^{\infty} \Gamma_{n} a_{n+p} z^{n+p} \quad(z \in U),
\end{aligned}
$$

where

$$
\Gamma_{n}=\frac{\left(a_{1}\right)_{n} \cdots\left(a_{q}\right)_{n}}{\left(b_{1}\right)_{n} \cdots\left(b_{s}\right)_{n}} \frac{1}{n !}, \quad(n \in \mathbb{N}) .
$$

To make the notation simple, we write

$$
H_{p, q, s}\left(a_{1}\right) f(z)=H_{p}\left(a_{1}, \ldots, a_{q} ; b_{1}, \ldots, b_{s}\right) f(z) .
$$

It easily follows from (9) or (10) that

$$
\begin{aligned}
z\left(H_{p, q, s}\right. & \left.\left(a_{1}\right) f(z)\right)^{\prime} \\
= & a_{1} H_{p, q, s}\left(a_{1}+1\right) f(z) \\
& -\left(a_{1}-p\right) H_{p, q, s}\left(a_{1}\right) f(z), \quad(z \in U) .
\end{aligned}
$$

It should be remarked that the linear operator $H_{p, q, s}\left(a_{1}\right)$ is a generalization of many other linear operators considered earlier. In particular, for $f \in A(p)$ we have the following observations:

(i) $H_{1,2,1}(a, b ; c) f(z)=\left(I_{c}^{a, b}\right) f(z)\left(a, b \in \mathbb{C} ; c \notin \mathbb{Z}_{0}^{-}\right)$, where the linear operator $I_{c}^{a, b}$ was investigated by Hohlov [6];

(ii) $H_{p, 2,1}(n+p, 1 ; 1) f(z)=D^{n+p-1} f(z)(n \in \mathbb{N} ; n>-p)$, where the linear operator $D^{n+p-1}$ was studied by Goel and Sohi [7]. In the case when $p=1, D^{n} f(z)$ is the Ruscheweyh derivative of $f(z)$ (see [8]);

(iii) $H_{p, 2,1}(\mu+p, 1 ; \mu+p+1) f(z)=J_{p, \delta}(f)(z)=((p+$ $\left.\delta) / z^{\delta}\right) \int_{0}^{z} t^{\delta-1} f(t) d t(\delta>-p)$, where $J_{p, \delta}$ is the generalized Bernardi-Libera-Livingston integral operator (see [9]);

(iv) $H_{p, 2,1}(p+1,1 ; p+1-\lambda) f(z)=\Omega_{z}^{(\lambda, p)} f(z)=(\Gamma(p+$ $1-\lambda) / \Gamma(p+1)) z^{\lambda} D_{z}^{\lambda} f(z)(-\infty \leq \lambda<p+1 ; z \in U)$, where $D_{z}^{\lambda} f(z)$ is the fractional integral of $f$ of order $-\lambda$ when $-\infty \leq \lambda<0$ and fractional derivative of $f$ of order $\lambda$ when $0 \leq \lambda<p+1$. The extended fractional differintegral operator $D_{z}^{(\lambda, p)}$ was introduced and studied by Patel and Mishra [10]. The fractional differential operator $\Omega_{z}^{(\lambda, p)}$ with $0 \leq \lambda<$ 1 was investigated by Srivastava and Aouf [11]. The operator $\Omega_{z}^{(\lambda, 1)}=\Omega_{z}^{\lambda}$ was introduced by Owa and Srivastava [12] (see also [13-15]). (v) $H_{\underline{p}, 2,1}(a, 1 ; c) f(z)=L_{p}(a, c) f(z)(a \in \mathbb{R} ; c \in \mathbb{R} \backslash$ $\left.\mathbb{Z}_{0}\right)$, where the linear operator $L_{p}(a, c)$ was studied by Saitoh [16] which yields the operator $L(a, c)$ introduced by Carlson and Shaffer [17] for $p=1$;

(vi) $H_{1,2,1}(\mu, 1 ; \lambda+1) f(z)=I_{\lambda, \mu} f(z)(\lambda>-1 ; \mu>0)$, where $I_{\lambda, \mu}$ is the Choi-Saigo-Srivastava operator [9] which is closely related to the Carlson-Shaffer [17] operator $L(\mu, \lambda+1) f(z)$;

(vii) $H_{p, 2,1}(p+1,1 ; n+p) f(z)=I_{n, p} f(z)(n \in \mathbb{Z} ; n>$ $-p)$, where the operator $I_{n, p}$ was considered by Liu and Noor [18];

(viii) $H_{p, 2,1}(\lambda+p, c ; a) f(z)=I_{p}^{\lambda}(a, c) f(z)(a, c \in \mathbb{R} \backslash$ $\left.\mathbb{Z}_{0}^{-} ; \lambda>-p\right)$, where $I_{p}^{\lambda}(a, c)$ is the Cho-KwonSrivastava operator [19].

In recent years, many interesting subclasses of analytic functions, associated with the Dziok-Srivastava operator $H_{p, q, s}\left(a_{1}\right)$ and its many special cases, were investigated by, for example, Dziok and Srivastava [5,20], Gangadharan et al. [21], Liu and Noor [18], Liu [22], Liu and Srivastava [23], and others (see also $[19,24-26]$ ). In the present paper, we shall use the method based upon the differential subordination to derive inclusion relationships and other interesting properties and characteristics of the Dziok-Srivastava operator $H_{p, q, s}\left(a_{1}\right)$.

\section{Main Results}

Unless otherwise mentioned, we assume throughout the sequel that $a_{i}>0 ; a_{i} \notin \mathbb{Z}_{0}^{-}(i=1, \ldots, q) ; \alpha>0 ; \mu>0$ and $-1 \leq B<A \leq 1$.

Let $P[k]$ denote the class of functions of the form

$$
\varphi(z)=1+c_{k} z^{k}+c_{k+1} z^{k+1}+\cdots
$$

that are analytic in $U$, we write $P[1]=P$. In our present investigation, we shall require the following lemmas.

Lemma 1 (see [2]). Let $h$ be analytic and convex (univalent) in $U$ with $h(0)=1$ and $\varphi \in P[k]$. If

$$
\varphi(z)+\frac{z \varphi^{\prime}(z)}{\gamma} \prec h(z),
$$

then, for $\gamma \neq 0$ and $\mathbb{R}(\gamma) \geq 0$,

$$
\varphi(z) \prec q(z)=\frac{\gamma}{k} z^{-\gamma / k} \int_{0}^{z} t^{\gamma / k-1} h(t) d t \prec h(z),
$$

and $q$ is the best dominant.

Lemma 2 (see [1]). Let $D$ be a set in the complex plane $\mathbb{C}$ and $b$ be a complex number satisfying $\mathbb{R}(b)>0$. Suppose that the function $\Psi: \mathbb{C}^{2} \times U \rightarrow \mathbb{C}$ satisfies the condition $\Psi(i x, y) \notin D$ for all real $x, y \leq-|b-i x| / 2 \mathbb{R}(b)$ and for all $z \in U$. If the functions $\varphi \in P$ and $\mathbb{R}\left\{\Psi\left(\varphi(z), z \varphi^{\prime}(z) ; z\right)\right\} \in D$, then $\mathbb{R}\{\varphi(z)\}>0$ in $U$. 
Lemma 3 (see [27]). Let $\phi$ be analytic in $U$ with $\phi(0)=1$ and $\phi(z) \neq 0$ for all $z \in U$. If there exist two points $z_{1}, z_{2} \in U$ such that

$$
-\frac{\pi}{2} \delta_{1}=\arg \left\{\phi\left(z_{1}\right)\right\}<\arg \{\phi(z)\}<\arg \left\{\phi\left(z_{2}\right)\right\}=\frac{\pi}{2} \delta_{2}
$$

for some $\delta_{1}$ and $\delta_{2}\left(\delta_{1}, \delta_{2}>0\right)$ and for all $z\left(|z|<\left|z_{1}\right|=\left|z_{2}\right|\right)$, then

$$
\begin{aligned}
& \frac{z_{1} \phi^{\prime}\left(z_{1}\right)}{\phi\left(z_{1}\right)}=-i\left(\frac{\delta_{1}+\delta_{2}}{2} m\right), \\
& \frac{z_{2} \phi^{\prime}\left(z_{2}\right)}{\phi\left(z_{2}\right)}=-i\left(\frac{\delta_{1}+\delta_{2}}{2} m\right),
\end{aligned}
$$

where

$$
m \geq \frac{1-|b|}{1+|b|}, \quad b=i \tan \left(\frac{\delta_{2}-\delta_{1}}{\delta_{2}+\delta_{1}}\right) .
$$

Theorem 4. Let $m \geq 1, \gamma>0$. Let $f \in A(k, p)$, then

$$
\begin{array}{r}
\mathbb{R}\left\{\frac{\left(H_{p, q, s}\left(a_{1}+1\right) f(z)\right)^{(j)}}{\left(H_{p, q, s}\left(a_{1}\right) f(z)\right)^{(j)}}\right\}<\frac{a_{1}+\gamma}{a_{1}} \\
(z \in U ; 0 \leq j<p),
\end{array}
$$

implies

$$
\begin{array}{r}
\mathbb{R}\left\{\left(\frac{\left(H_{p, q, s}\left(a_{1}+1\right) f(z)\right)^{(j)}}{z^{p^{-j}}}\right)^{-1 / 2 \gamma m}\right\}>2^{-1 / m} \\
(z \in U ; 0 \leq j<p) .
\end{array}
$$

The bound $2^{-1 / m}$ is the best possible.

Proof. It easily follows from (13) that

$$
\begin{aligned}
z\left(H_{p, q, s}\left(a_{1}\right) f(z)\right)^{(j+1)} & \\
= & a_{1}\left(H_{p, q, s}\left(a_{1}+1\right) f(z)\right)^{(j)} \\
& -\left(a_{1}-p+j\right)\left(H_{p, q, s}\left(a_{1}\right) f(z)\right)^{(j)} \\
& (z \in U ; 0 \leq j<p) .
\end{aligned}
$$

From (20) and (22), we have

$$
\begin{array}{r}
\mathbb{R}\left\{\frac{z\left(H_{p, q, s}\left(a_{1}+1\right) f(z)\right)^{(j+1)}}{\left(H_{p, q, s}\left(a_{1}\right) f(z)\right)^{(j)}}\right\}<\gamma+p-j \\
(z \in U ; 0 \leq j<p) .
\end{array}
$$

That is,

$$
\begin{aligned}
& -\frac{1}{2 \gamma}\left(\frac{z\left(H_{p, q, s}\left(a_{1}+1\right) f(z)\right)^{(j+1)}}{\left(H_{p, q, s}\left(a_{1}\right) f(z)\right)^{(j)}}-p+j\right) \\
& \quad \prec \frac{z}{1-z} \quad(z \in U) .
\end{aligned}
$$

Let

$$
\varphi(z)=\left(\frac{(p-j) !}{p !} \frac{\left(H_{p, q, s}\left(a_{1}\right) f(z)\right)^{(j)}}{z^{p-j}}\right)^{-1 / 2 \gamma} \quad(z \in U),
$$

then (24) may be written as

$$
z(\log \varphi(z))^{\prime} \prec z\left(\log \frac{1}{1-z}\right)^{\prime}
$$

By using a well-known result (see [28]) to (26) we obtain that

$$
\varphi(z) \prec \frac{1}{1-z},
$$

or, equivalently,

$$
\begin{gathered}
\left(\frac{(p-j) !}{p !} \frac{\left(H_{p, q, s}\left(a_{1}\right) f(z)\right)^{(j)}}{z^{p-j}}\right)^{-1 / 2 \gamma m} \\
=\left(\frac{1}{1-\omega(z)}\right)^{1 / m}
\end{gathered}
$$

where $\omega$ is analytic in $U, \omega(0)=0$ and $|\omega(z)|<1$ for $z \in U$. Since $\mathbb{R}\left(t^{1 / m}\right) \geq(\mathbb{R}(t))^{1 / m}$ for $\mathbb{R}(t)>0$ and $m \geq 1$, (28) yields

$$
\begin{aligned}
& \mathbb{R}\left(\frac{(p-j) !}{p !} \frac{\left(H_{p, q, s}\left(a_{1}\right) f(z)\right)^{(j)}}{z^{p-j}}\right)^{-1 / 2 \gamma m} \\
& \quad \geq\left(\mathbb{R}\left(\frac{1}{1-\omega(z)}\right)\right)^{1 / m} \geq 2^{-1 / m} \quad(z \in U) .
\end{aligned}
$$

To see that the bound $2^{-1 / m}$ cannot be increased, we consider the function

$$
\begin{array}{r}
g(z)=z^{p}+\frac{p !}{(p-j) !} \sum_{n=1}^{\infty} \frac{(-2 \gamma)_{n}(n+p-j) !}{n !(n+p) ! \Gamma_{n}} z^{n+p} \\
(z \in U) .
\end{array}
$$

Since

$$
\frac{(p-j) !}{p !} \frac{\left(H_{p, q, s}\left(a_{1}\right) g(z)\right)^{(j)}}{z^{p-j}}=(1-z)^{-2 \gamma},
$$

we easily have that $g$ satisfies (20) and

$$
\mathbb{R}\left(\frac{(p-j) !}{p !} \frac{\left(H_{p, q, s}\left(a_{1}\right) g(z)\right)^{(j)}}{z^{p^{-j}}}\right)^{-1 / 2 \gamma m} \longrightarrow 2^{-1 / m}
$$

as $\mathbb{R}(z)=z \rightarrow 1^{-}$. This completes the proof of Theorem 4. 
Theorem 5. Let $\alpha \geq 0, \gamma>1$. If $f \in A(p)$ satisfies the following inequality

$$
\begin{array}{r}
\mathbb{R}\left\{(1-\alpha) \frac{\left(H_{p, q, s}\left(a_{1}+1\right) f(z)\right)^{(j)}}{\left(H_{p, q, s}\left(a_{1}\right) f(z)\right)^{(j)}}\right. \\
\left.+\alpha \frac{\left(H_{p, q, s}\left(a_{1}+2\right) f(z)\right)^{(j)}}{\left(H_{p, q, s}\left(a_{1}+1\right) f(z)\right)^{(j)}}\right\}<\gamma \\
(0 \leq j<p ; z \in U),
\end{array}
$$

then

$$
\mathbb{R}\left\{\frac{\left(H_{p, q, s}\left(a_{1}+1\right) f(z)\right)^{(j)}}{\left(H_{p, q, s}\left(a_{1}\right) f(z)\right)^{(j)}}\right\}<\beta \quad(0 \leq j<p ; z \in U),
$$

where $\beta \in(1, \infty)$ is the positive root of the equation

$$
2\left(a_{1}-\alpha+1\right) x^{2}+(3 \alpha-2 \gamma \alpha-2 \gamma) x-\alpha=0 .
$$

Proof. Let

$$
\varphi(z)=\frac{1}{\beta-1}\left[\beta-\frac{\left(H_{p, q, s}\left(a_{1}+1\right) f(z)\right)^{(j)}}{\left(H_{p, q, s}\left(a_{1}\right) f(z)\right)^{(j)}}\right] \quad(z \in U),
$$

then $\varphi(z)$ is analytic in $U$ and $\varphi(0)=1$. Differentiating (36) and using (22), we obtain that

$$
\begin{aligned}
(1-\alpha) & \frac{\left(H_{p, q, s}\left(a_{1}+1\right) f(z)\right)^{(j)}}{\left(H_{p, q, s}\left(a_{1}\right) f(z)\right)^{(j)}} \\
+ & \alpha \frac{\left(H_{p, q, s}\left(a_{1}+2\right) f(z)\right)^{(j)}}{\left(H_{p, q, s}\left(a_{1}+1\right) f(z)\right)^{(j)}} \\
= & \beta-\frac{\alpha(\beta-1)}{a_{1}+1}-\frac{\left(a_{1}-\alpha+1\right)(\beta-1)}{a_{1}+1} \varphi(z) \\
& -\frac{\alpha(\beta-1)}{a_{1}+1} \frac{z \varphi^{\prime}(z)}{\beta-(\beta-1) \varphi(z)} \\
= & \psi\left(\varphi(z), z \varphi^{\prime}(z)\right),
\end{aligned}
$$

where

$$
\begin{aligned}
\psi(r, s)= & -\frac{\alpha(\beta-1)}{a_{1}+1}-\frac{\left(a_{1}-\alpha+1\right)(\beta-1)}{a_{1}+1} r \\
& -\frac{\alpha(\beta-1)}{a_{1}+1} \frac{s}{\beta-(\beta-1) r} .
\end{aligned}
$$

Using (33) and (38), we have

$$
\left\{\psi\left(\varphi(z), z \varphi^{\prime}(z)\right): z \in U\right\} \subset D=\{z \in \mathbb{C}: \mathbb{R}(z)<\gamma\} .
$$

Now for all real $x, y \leq-\left(1+x^{2}\right) / 2$, we have

$$
\begin{aligned}
\mathbb{R}\{\psi(i x, y)\} & =\beta-\frac{\alpha(\beta-1)}{a_{1}+1}-\frac{\alpha(\beta-1)}{a_{1}+1} \frac{\beta y}{\beta^{2}+(\beta-1)^{2} x^{2}} \\
& \geq \beta-\frac{\alpha(\beta-1)}{a_{1}+1}+\frac{\alpha \beta(\beta-1)}{2\left(a_{1}+1\right)} \frac{1+x^{2}}{\beta^{2}+(\beta-1)^{2} x^{2}} \\
& \geq \beta-\frac{\alpha(\beta-1)}{a_{1}+1}+\frac{\alpha(\beta-1)}{2 \beta\left(a_{1}+1\right)} \\
& =\beta-\frac{\alpha(\beta-1)(2 \beta-1)}{2 \beta\left(a_{1}+1\right)}=\gamma,
\end{aligned}
$$

where $\beta$ is the positive root of (35).

Note that for $\alpha \geq 0, \gamma>1, a_{1}>0$ and

$$
h(x)=2\left(a_{1}-\alpha+1\right) x^{2}+(3 \alpha-2 \gamma \alpha-2 \gamma) x-\alpha,
$$

we have $h(0)=-\alpha \leq 0$ and $h(1)=2 a_{1}(1-\gamma)-2 \gamma<0$. This shows $\beta \in(0,+\infty)$. Hence for each $z \in U, \psi(i x, y) \notin \Omega$. By Lemma 2, we get $\mathbb{R}\{\varphi(z)\}>0(z \in U)$, and this proves (34).

Theorem 6. Suppose that $0 \leq j<p ; \alpha>0$ and $0<\delta_{1}, \delta_{2} \leq 1$. If $F_{\alpha}$ given by

$$
\begin{aligned}
F_{\alpha}(z)= & \left(1-\alpha-\alpha a_{1}+\alpha p\right) H_{p, q, s}\left(a_{1}\right) f(z) \\
& +\alpha a_{1} H_{p, q, s}\left(a_{1}+1\right) f(z)
\end{aligned}
$$

satisfies

$$
-\frac{\pi}{2} \delta_{1}<\arg \left\{\frac{F_{\alpha}^{(j)}(z)}{z^{p-j}}\right\}<\frac{\pi}{2} \delta_{2} \quad(z \in U),
$$

then

$$
-\frac{\pi}{2} \eta_{1}<\arg \left\{\frac{\left(H_{p, q, s}\left(a_{1}\right) f(z)\right)^{(j)}}{z^{p-j}}\right\}<\frac{\pi}{2} \eta_{2} \quad(z \in U),
$$

where $\eta_{1}$ and $\eta_{2}$ are the solution of the equations:

$$
\begin{aligned}
& \delta_{1}=\eta_{1}+\frac{2}{\pi} \arctan \left[\frac{\alpha\left(\eta_{1}+\eta_{2}\right)}{2(1-\alpha+\alpha p)}\left(\frac{1-|b|}{1+|b|}\right)\right], \\
& \delta_{2}=\eta_{2}+\frac{2}{\pi} \arctan \left[\frac{\alpha\left(\eta_{1}+\eta_{2}\right)}{2(1-\alpha+\alpha p)}\left(\frac{1-|b|}{1+|b|}\right)\right],
\end{aligned}
$$

where $b$ is given by (19).

Proof. Using (42) and the identity (22), it follows that

$$
\begin{aligned}
F_{\alpha}^{(j)}(z)= & (1-\alpha+\alpha j)\left(H_{p, q, s}\left(a_{1}\right) f(z)\right)^{(j)} \\
& +\alpha z\left(H_{p, q, s}\left(a_{1}\right) f(z)\right)^{(j+1)},
\end{aligned}
$$


for $0 \leq j<p$. Putting

$$
\varphi(z)=\frac{(p-j) !}{p !} \frac{\left(H_{p, q, s}\left(a_{1}\right) f(z)\right)^{(j)}}{z^{p^{-j}}} \quad(z \in U) .
$$

On differentiating (47) followed by a simple calculation, we get

$$
\begin{aligned}
\frac{F_{\alpha}^{(j)}(z)}{z^{p-j}}= & \frac{p !(1-\alpha+\alpha p)}{(p-j) !} \\
& \times\left\{\varphi(z)+\frac{\alpha}{1-\alpha+\alpha p} z \varphi^{\prime}(z)\right\} \quad(z \in U) .
\end{aligned}
$$

Let $h$ be the function which maps $U$ onto the angular domain $\left\{w \in \mathbb{C}:-(\pi / 2) \delta_{1}<\arg \{w\}<(\pi / 2) \delta_{2}\right\}$ with $h(0)=1$. By using (43) in (48), we get

$$
\varphi(z)+\frac{\alpha}{1-\alpha+\alpha p} z \varphi^{\prime}(z) \prec h(z) .
$$

Further, an application of Lemma 1 yields $\mathbb{R}\{\varphi(z)\}>0$ in $U$ and hence $\varphi(z) \neq 0$ for $z \in U$.

Suppose there exist two points $z_{1}, z_{2} \in U$ such that the condition (28) is satisfied. Then by Lemma 3, we obtain (18) under the constraint (19). Therefore, we have

$$
\begin{aligned}
& \arg \{\left.(1-\alpha+\alpha p) \varphi\left(z_{1}\right)+\alpha z \varphi^{\prime}\left(z_{1}\right)\right\} \\
&= \arg \left\{\varphi\left(z_{1}\right)\right\}+\arg \left\{(1-\alpha+\alpha p)+\alpha \frac{z_{1} \varphi^{\prime}\left(z_{1}\right)}{\varphi\left(z_{1}\right)}\right\} \\
&=-\frac{\pi}{2} \eta_{1}+\arg \left\{(1-\alpha+\alpha p)-i \frac{\alpha\left(\eta_{1}+\eta_{2}\right)}{2} m\right\} \\
&=-\frac{\pi}{2} \eta_{1}-\arctan \left\{\frac{\alpha\left(\eta_{1}+\eta_{2}\right)}{2(1-\alpha+\alpha p)} m\right\} \\
& \leq-\frac{\pi}{2} \eta_{1}-\arctan \left\{\frac{\alpha\left(\eta_{1}+\eta_{2}\right)}{2(1-\alpha+\alpha p)}\left(\frac{1-|b|}{1+|b|}\right)\right\}, \\
& \arg \left\{(1-\alpha+\alpha p) \varphi\left(z_{2}\right)+\alpha z \varphi^{\prime}\left(z_{2}\right)\right\} \\
& \geq-\frac{\pi}{2} \eta_{2}-\arctan \left\{\frac{\alpha\left(\eta_{1}+\eta_{2}\right)}{2(1-\alpha+\alpha p)}\left(\frac{1-|b|}{1+|b|}\right)\right\}
\end{aligned}
$$

which contradicts the assumption (43). This proves the assertion (44) of the Theorem 6.

For $\delta_{1}=\delta_{2}=\delta$, Theorem 6 reduces to the following corollary.

Corollary 7. Suppose that $0 \leq j<p$ and $\alpha>0$. If $F_{\alpha}$ defined by (42) satisfies

$$
\left|\arg \left\{\frac{F_{\alpha}^{(j)}(z)}{z^{p^{-j}}}\right\}\right|<\frac{\pi}{2} \delta \quad(0<\delta \leq 1 ; z \in U),
$$

then

$$
\left|\arg \left\{\frac{\left(H_{p, q, s}\left(a_{1}\right) f(z)\right)^{(j)}}{z^{p^{-j}}}\right\}\right|<\frac{\pi}{2} \eta \quad(z \in U),
$$

where $\eta(0<\eta \leq 1)$ is the solution of the equation:

$$
\delta=\eta+\frac{2}{\pi} \arctan \left(\frac{\alpha \eta}{1-\alpha+\alpha p}\right) .
$$

\section{References}

[1] S. S. Miller and P. T. Mocanu, "Differential subordinations and univalent functions," The Michigan Mathematical Journal, vol. 28, no. 2, pp. 157-172, 1981.

[2] S. S. Miller and P. T. Mocanu, Differential Subordinations: Theory and Applications, vol. 225 of Monographs and Textbooks in Pure and Applied Mathematics, Marcel Dekker, New York, NY, USA, 2000.

[3] S. S. Miller and P. T. Mocanu, "Subordinants of differential superordinations," Complex Variables, vol. 48, no. 10, pp. 815-826, 2003.

[4] H. M. Srivastava and P. W. Karlsson, Multiple Gaussian Hypergeometric Series, Ellis Horwood, Chichester, UK, 1985.

[5] J. Dziok and H. M. Srivastava, "Classes of analytic functions associated with the generalized hypergeometric function," Applied Mathematics and Computation, vol. 103, no. 1, pp. 1-13, 1999.

[6] Yu. E. Hohlov, "Operators and operations in the class of univalent functions," Izvestiya Vysšhikh Učhebnykh Zavedenii. Matematika, vol. 10, pp. 83-89, 1978 (Russian).

[7] R. M. Goel and N. S. Sohi, "A new criterion for $p$-valent functions," Proceedings of the American Mathematical Society, vol. 78, no. 3, pp. 353-357, 1980.

[8] S. Ruscheweyh, "New criteria for univalent functions," Proceedings of the American Mathematical Society, vol. 49, pp. 109-115, 1975.

[9] J. H. Choi, M. Saigo, and H. M. Srivastava, "Some inclusion properties of a certain family of integral operators," Journal of Mathematical Analysis and Applications, vol. 276, no. 1, pp. 432-445, 2002.

[10] J. Patel and A. K. Mishra, “On certain subclasses of multivalent functions associated with an extended fractional differintegral operator," Journal of Mathematical Analysis and Applications, vol. 332, no. 1, pp. 109-122, 2007.

[11] H. M. Srivastava and M. K. Aouf, "A certain fractional derivative operator and its applications to a new class of analytic and multivalent functions with negative coefficients I and II," Journal of Mathematical Analysis and Applications, vol. 171, no. 1, pp. 1-13, 1992, ibid, vol. 192, pp. 673-688, 1995.

[12] S. Owa and H. M. Srivastava, "Univalent and starlike generalized hypergeometric functions," Canadian Journal of Mathematics, vol. 39, no. 5, pp. 1057-1077, 1987.

[13] J. Dziok, "Classes of analytic functions involving some integral operator," Folia. Scientiarum Universitatis Technicae Resoviensis, no. 20, pp. 21-39, 1996.

[14] J. L. Liu and J. Patel, "Certain properties of multivalent functions associated with an extended fractional differintegral operator," Applied Mathematics and Computation, vol. 203, no. 2, pp. 703-713, 2008. 
[15] S. Owa, "On the distortion theorems. I," Kyungpook Mathematical Journal, vol. 18, no. 1, pp. 53-59, 1978.

[16] H. Saitoh, "A linear operator and its applications of first order differential subordinations," Mathematica Japonica, vol. 44, no. 1, pp. 31-38, 1996.

[17] B. C. Carlson and D. B. Shaffer, "Starlike and prestarlike hypergeometric functions," SIAM Journal on Mathematical Analysis, vol. 15, no. 4, pp. 737-745, 1984.

[18] J.-L. Liu and K. I. Noor, "Some properties of Noor integral operator," Journal of Natural Geometry, vol. 21, no. 1-2, pp. 81-90, 2002.

[19] N. E. Cho, O. S. Kwon, and H. M. Srivastava, "Inclusion relationships and argument properties for certain subclasses of multivalent functions associated with a family of linear operators," Journal of Mathematical Analysis and Applications, vol. 292, no. 2, pp. 470-483, 2004.

[20] J. Dziok and H. M. Srivastava, "Certain subclasses of analytic functions associated with the generalized hypergeometric function," Integral Transforms and Special Functions, vol. 14, no. 1, pp. 7-18, 2003.

[21] A. Gangadharan, T. N. Shanmugam, and H. M. Srivastava, "Generalized hypergeometric functions associated with Kuniformly convex functions," Computers and Mathematics with Applications, vol. 44, no. 12, pp. 1515-1526, 2002.

[22] J.-L. Liu, "Strongly starlike functions associated with the Dziok-Srivastava operator," Tamkang Journal of Mathematics, vol. 35, p. 37-42, 2004.

[23] J. L. Liu and H. M. Srivastava, "Certain properties of the DziokSrivastava operator," Applied Mathematics and Computation, vol. 159, no. 2, pp. 485-493, 2004.

[24] K. I. Noor, "Some classes of $p$-valent analytic functions defined by certain integral operator," Applied Mathematics and Computation, vol. 157, no. 3, pp. 835-840, 2004.

[25] K. I. Noor and M. A. Noor, "On Integral Operators," Journal of Mathematical Analysis and Applications, vol. 238, no. 2, pp. 341-352, 1999.

[26] H. M. Srivastava and J. Patel, "Some subclasses of multivalent functions involving a certain linear operator," Journal of Mathematical Analysis and Applications, vol. 310, no. 1, pp. 209-228, 2005.

[27] N. Takahashi and M. Nunokawa, "A certain connection between starlike and convex functions," Applied Mathematics Letters, vol. 16, no. 5, pp. 653-655, 2003.

[28] T. J. Suffridge, "Some remarks on convex maps of the unit disk," Duke Mathematical Journal, vol. 37, pp. 775-777, 1970. 


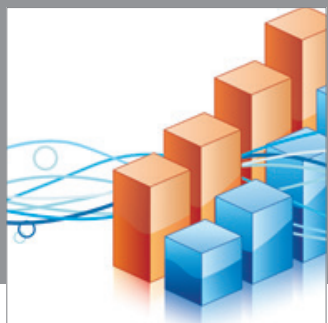

Advances in

Operations Research

mansans

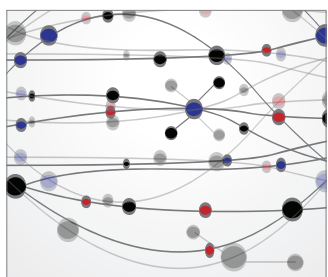

The Scientific World Journal
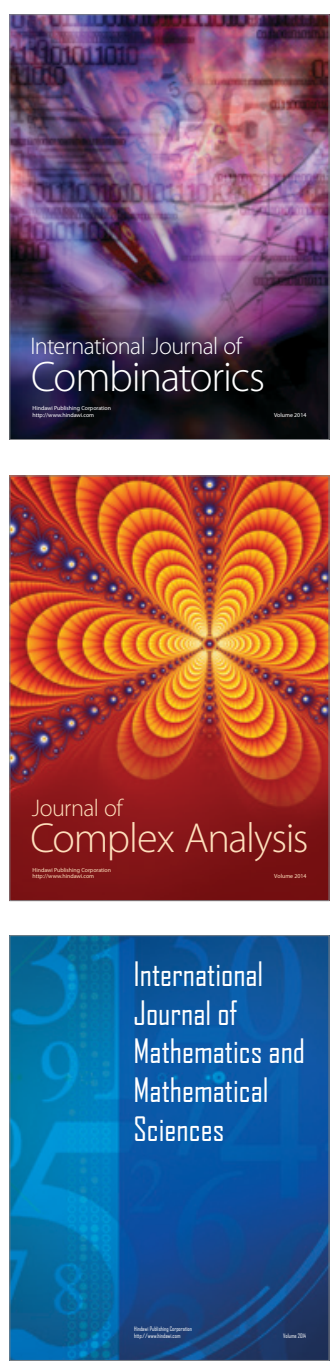
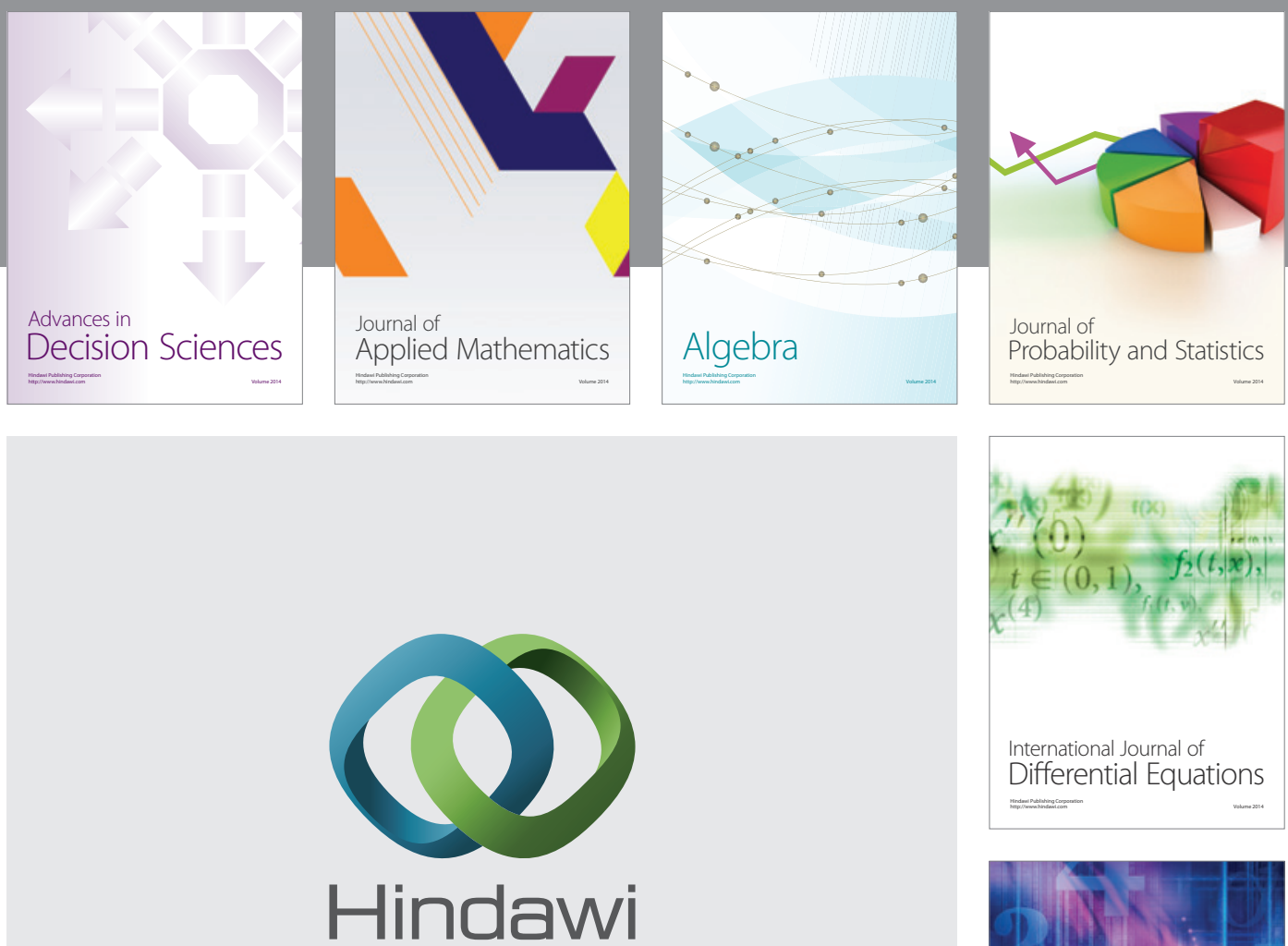

Submit your manuscripts at http://www.hindawi.com
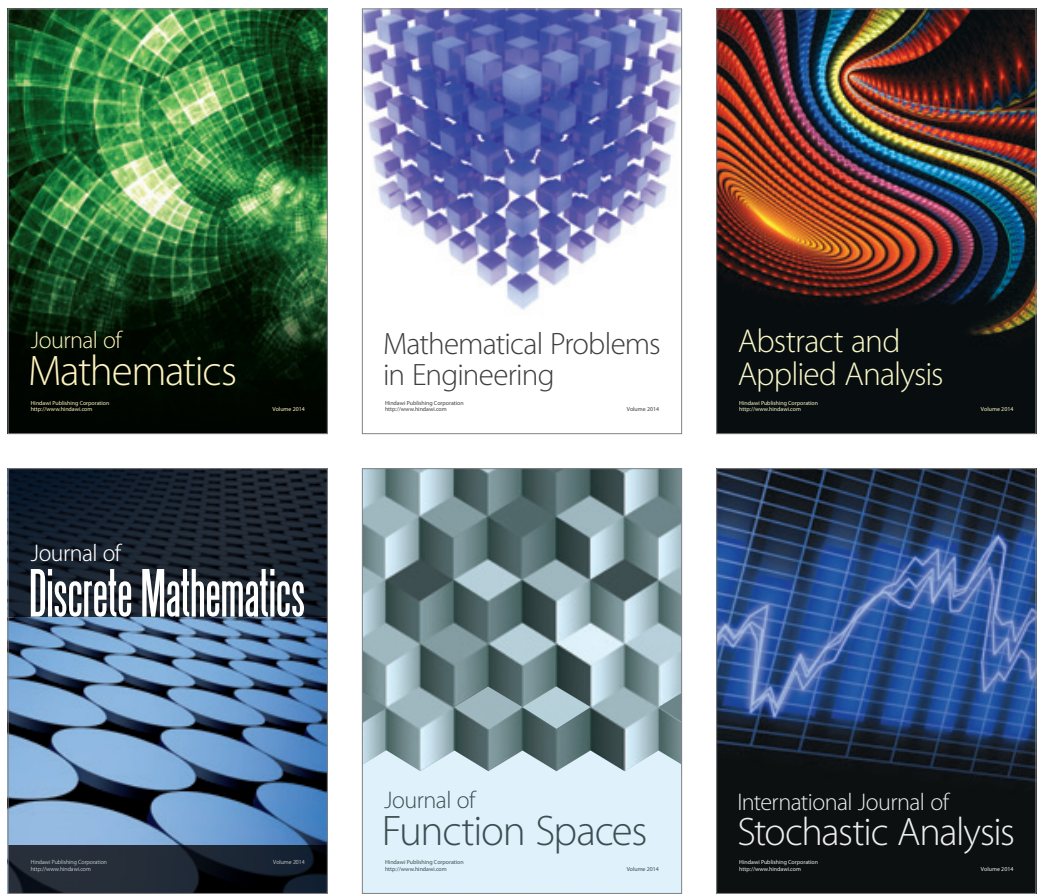

Journal of

Function Spaces

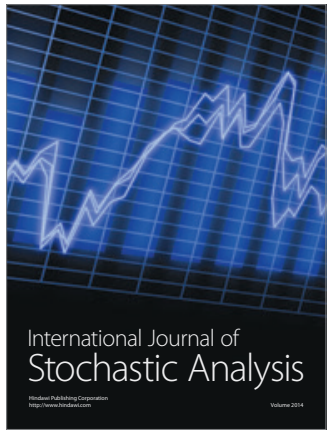

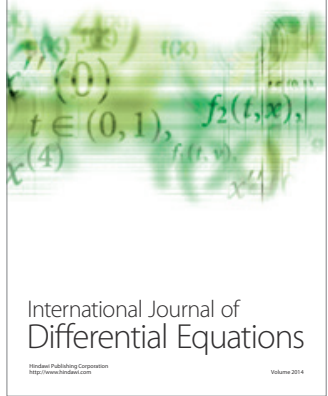
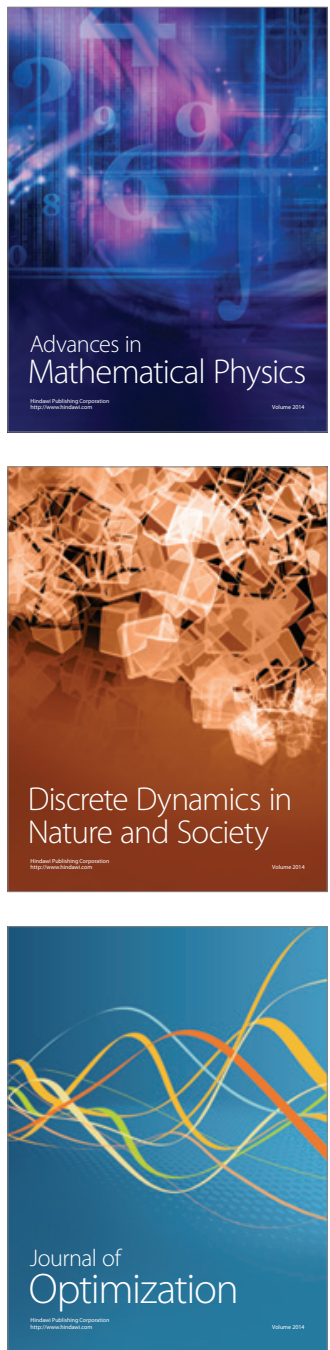